Metastasis from serous carcinoma of the ovary usually occurs in the subdiaphragmatic region. Metastasis to the breast and/or axillary-intramammary lymph node is very rare. It usually occurs in advanced disease, and it is important to distinguish a primary breast cancer from an ovarian cancer metastasis since the management of these two entities is totally different. Here we present a patient with metastasis to the breast and intramammary lymph nodes from ovarian serous carcinoma 25 months after diagnosis.

Key words: ovarian cancer, intramammary, lymph node, metastasis.

\section{Intramammary lymph node metastasis in a patient with ovarian carcinoma and a brief review of the literature}

\author{
Lutfiye Demir ${ }^{1}$, Cigdem Erten ${ }^{1}$, Seyran Ceri Yigit ${ }^{2}$, Alper Can ${ }^{1}$, Ahmet Dirican ${ }^{1}$, \\ Vedat Bayoglu ${ }^{1}$, Yuksel Kucukzeybek ${ }^{1}$, Isil Somali ${ }^{1}$, M Oktay Tarhan $^{1}$
}

${ }^{1}$ Clinic of Medical Oncology, Izmir Ataturk Training and Research Hospital, Izmir, Turkey 2Department of Pathology, Izmir Ataturk Training and Research Hospital, Izmir, Turkey

\section{Introduction}

Ovarian cancer is the most common cause of death among women with gynecologic malignancies and the fifth leading cause of cancer death in women in the United States [1]. Approximately 21550 new cases and 14600 deaths are estimated annually in the US [1]. There are, however, large variations in the incidence of ovarian cancer in different areas of the world. The highest incidence areas are in Europe (especially Austria, Germany and the United Kingdom) and North America [2-4]. The estimated number of newly diagnosed cases in the European Union was 42700 in 2004 with a mortality of 12/100 000 women/year. Metastasis of ovarian serous carcinoma to the breast and axillary lymph nodes is very rare and usually occurs very late in the course of the disease $[5,6]$. Therefore, metastatic cancer to the breast from an ovarian primary is associated with shortened survival, most of the patients dying a few months after the metastasis. Karam et al. [7] reported a retrospective analysis of 29 patients with epithelial ovarian cancer and breast/axillary mass from 1990 to 2007. Ten of them were metastatic to breast, and 19 patients had secondary primary carcinoma of the breast. The mean disease-free survival (DFS) was 14.9 versus 77.4 months for patients with epithelial ovarian cancer metastatic to breast and for patients with secondary primary breast cancer respectively. Thus, it is important to distinguish primary breast cancer from ovarian cancer metastasis.

\section{Case report}

A 52-year-old woman with complaints of inguinal tenderness and increased abdominal size for three months was admitted to our hospital, Izmir Ataturk Training and Research Hospital, Department of Gynecology, in September 2006. An irregular, fixed pelvic mass and ascites were detected in her physical and pelvic examination. She underwent an abdominal ultrasound examination, abdominopelvic computerized tomography (CT), and a plain chest radiography. These imaging studies demonstrated ascites, bilateral adnexal masses, and a right-sided minimal pleural effusion. Serum CA125 level was elevated (4550 $\mathrm{U} / \mathrm{ml}$ ). After preoperative evaluation, the patient underwent laparotomy and an optimal cytoreduction procedure with total abdominal hysterectomy, bilateral salpingo-oophorectomy, omentectomy, and lymphadenectomy. An $8 \mathrm{~cm}$ diameter tumor on the right ovary and a $10 \mathrm{~cm}$ tumor on the left ovary and 2 liters of ascites were detected. Peritoneal washing cytology was malignant. The frozen and final pathology was compatible with well differentiated, malignant serous carcinoma (Fig. 1), with invasion of the omentum, peritoneum, and uterine wall (stage IIIC). Postoperative CA125 level was $2177 \mathrm{U} / \mathrm{ml}$. Immediate postoperative CT scan showed a solid 4-5 sized mass in the right inguinal region, and multiple millimetric lymph nodes.

She received a combination therapy of carboplatin ( $A \cup C=6$, d1 of a 21-day cycle) and paclitaxel (175 mg/m², on day 1 of a 21-day cycle) which was initi- 
ated in December 2006. After 6 chemotherapy cycles, in April 2007, serum CA 125 level was $860 \mathrm{U} / \mathrm{ml}$ without any radiological improvement. Then she was treated with a combination therapy of liposomal doxorubicin $\left(30 \mathrm{mg} / \mathrm{m}^{2}\right.$, on day 1 of a 21 -day cycle) and gemcitabine $\left(1000 \mathrm{mg} / \mathrm{m}^{2}\right.$, on days 1 and 8 of a 21-day cycle). The treatment was continued for 14 courses with a remarkable CA- 125 response. The chemotherapy was completed in July 2008 and serum CA125 level was $95.5 \mathrm{U} / \mathrm{ml}$ at that time. The solid size of the solid mass in the right inguinal region was same, but lymph nodes were not seen in the follow-up CT of the abdomen/pelvis. Surgery was not planned because it was considered as a postoperative benign lesion by her gynecologist. Five months later, in the thorax CT, a $2 \mathrm{~cm}$-sized lymph node in right axillary fossa was seen. The mammography showed a $9 \mathrm{~mm}$ sized opacity without malignant features in the right breast. During the follow-up, in January 2009, the serum CA125 level increased to $302 \mathrm{U} / \mathrm{ml}$. For that reason, oral etoposide (100 mg/day, 14/28 day) was started. After 6 cycles, the CA125 level increased to $914 \mathrm{U} / \mathrm{ml}$ and the axillary lymph node and intramammary lesion showed progression. Surgical biopsy of the intramammary lymph node was compatible with metastasis of ovarian serous carcinoma (Fig. 2). The immunoperoxidase study for WT-1 was positive (Fig. 3). Following this diagnosis, a combination of carboplatin $(A \cup C=6$, on day 1 of a 21-day cycle) and cyclophosphamide $\left(750 \mathrm{mg} / \mathrm{m}^{2}\right.$, on day 1 of a 21-day cycle) was started in December 2009. After 6 cycles, the number and size of axillary and intramammary lymph nodes increased and newly appearing intra-abdominal lymph nodes were seen in the CT scan. After then, the patient was treated with topotecan $\left(4 \mathrm{mg} / \mathrm{m}^{2}\right.$, weekly), however she progressed after 4 months, and paclitaxel ( $80 \mathrm{mg} / \mathrm{m}^{2}$, weekly) was treated with. She has been still treated by weekly paclitaxel for 4 months and has a stable radiological and clinical response.

\section{Discussion}

Ovarian carcinoma is the second most common gynecologic malignancy. Approximately 21550 women in the United States are diagnosed with ovarian cancer annually, and an estimated 15520 women die of the disease [1]. Papillary serous histology accounts for as many as $75 \%$ of them [8]. Metastasis of ovarian serous carcinoma to the breast and axillary lymph nodes is very rare [5, 6]. Only a few cases have been reported in the literature so far. Moreira et al. [9] reported a 29-year-old woman who presented with an intramammary lymph node metastasis of a borderline papillary ovarian tumor which had been resected a year before diagnosis. Recine et al. [5] reported a series of 14 cases of ovarian serous carcinoma which had metastases to breast and axillary lymph nodes. One of them was stage I at the initial diagnosis while 10 of them were stage III and 3 of them were stage IV at the time of the diagnosis. Only one patient had stage IV disease initially with both axillary lymph node metastasis and breast lesion. The breast and/or axillary lymph node metastases were discovered at an average of 30 months after presentation (in patients with stage III). Another three case were reported by Micha et al. [10] in 2006. All of them had stage III disease and presented with a mass in the breast. One of the cases presented an inflammatory pattern.

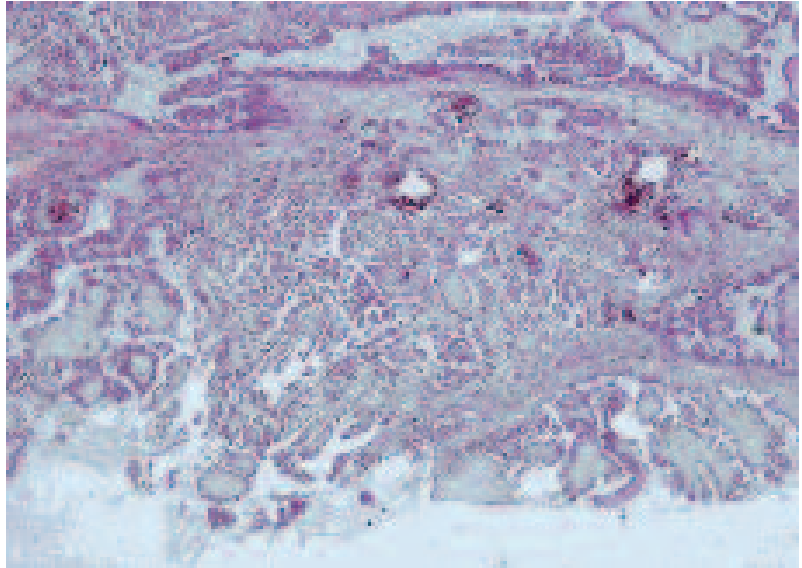

Fig. 1. Malignant serous tumor of the ovary

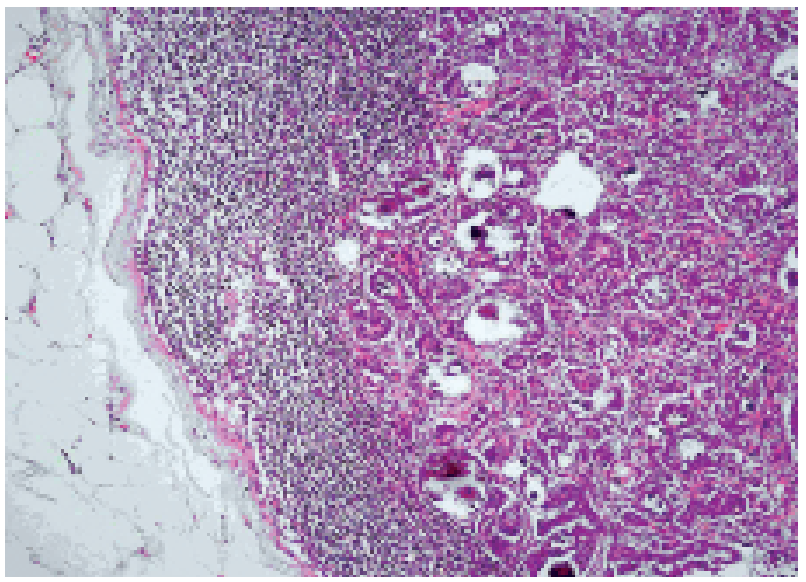

Fig. 2. Metastatic ovarian serous carcinoma in the mammary lymph node

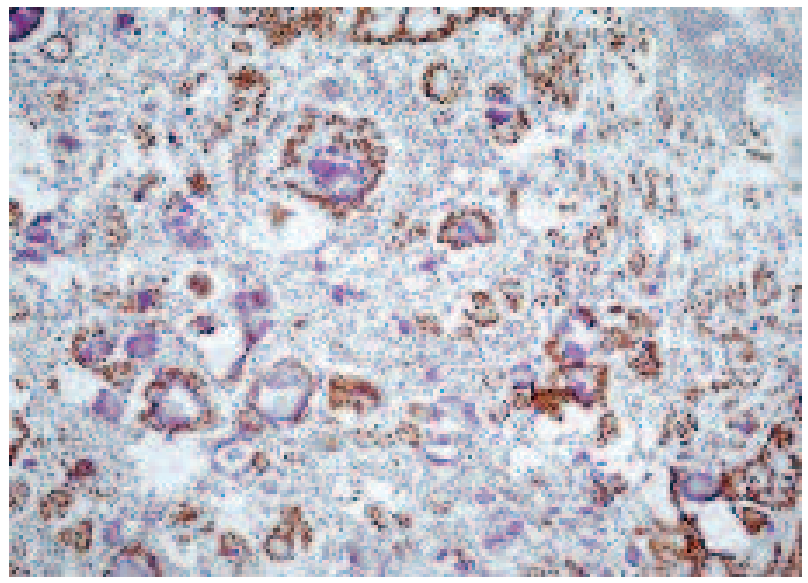

Fig. 3. WT-1 positivity (seen as brown) in metastatic serous ovarian cancer cells

Metastatic cancer to the breast from an ovarian primary is associated with a poor prognosis, with a majority of patients dying within one year [6, 11]. Kayikcioglu et al. [12] presented the case of a patient that was initially stage IIC epithelial ovarian cancer. After two years, she presented with bilateral metastatic breast disease derived from the primary ovarian cancer. Although she was heavily treated, she died 18 months after diagnosis. 
Differentiation between metastatic and primary tumors of the breast is of great importance because treatment and prognosis differ significantly. Clinical history, the presence of papillary architecture, and immunohistochemical features are important in establishing the correct diagnosis. WT-1, the Wilms tumor gene product, can be expressed in some types of ovarian tumors, other solid tumors (such as stomach, prostate, biliary and urinary system tumors, malignant melanoma) and leukemias. Most of the studies showed that serous carcinomas are usually positive and endometrioid ovarian cancers are usually negative for this marker [13] Yamamoto et al. [14] analyzed WT-1 immunoreactivity in 119 patients with ovarian serous cancer and showed that WT-1 positivity is positively correlated with high grade, advanced stage and higher Ki-67 labeling index, higher bcl-2 expression and poorer outcome in a study. Although WT-1 positivity can be seen in many solid tumors, it has been identified as a possible marker mostly for ovarian cancer. A study of 117 patients involving primary breast cancer, breast cancer metastatic to ovary, and primary ovarian cancer showed that $76 \%$ of primary ovarian cancer patients were positive for WT-1, but none of the patients with breast cancer showed WT-1 positivity [15]. Gross cystic disease fluid protein (GCDFP-15) and mammaglobin are both widely used and accepted markers for epithelia of breast origin. Cytoplasmic expression of GCDFP-15 and mammaglobin was observed in respectively $73.3 \%$ and $72.1 \%$ of invasive breast carcinomas in a study [16]. These two markers were studied in paraffin-embedded tissues of the patients with primary breast cancer, serous carcinoma (ovarian and uterine), and metastatic breast cancer to the ovary. Mammaglobin was negative in all serous carcinomas, while it was more frequently and diffusely expressed in breast carcinomas when compared to GCDFP-15 (14/21 patients vs. 8/21 patients for GCDFP-15) [17]. Chia et al. compared the diagnostic utility of these two markers in confirming a breast origin for recurrent tumors in a recent study; they demonstrated that metastases to the breast from other organs and metastatic lesions from non-breast primaries were uniformly negative for both mammaglobin and GCDFP-15, and also mammaglobin was superior to GCDFP-15 in detecting a tumor of breast origin [18]. As a result, in addition to papillary morphology, metastatic breast lesions from ovarian serous tumor origin are usually negative for GCDFP15 and mammaglobin and positive for WT-1. We established the diagnosis of metastasis from ovarian cancer by using WT1 immunoreactivity and typical papillary architecture of serous cancer cells in this case.

The present patient had a primary ovarian carcinoma and had been heavily treated with multiple chemotherapy regimens. She developed an intramammary and axillary lymph node metastasis 25 months after diagnosis. Serous carcinoma cells and WT-1 positivity confirmed metastasis of ovarian cancer. The patient has been receiving a sixth-line chemotherapy protocol and she is still alive 29 months after intramammary lymph node metastasis was detected. Although she has an advanced disease, she has no visceral organ metastasis and she has in total a 56-month survival time since diagnosis. This shows that survival could differ from the other patients discussed above. Therefore each patient should be managed individually.

\section{References}

1. Jemal A, Siegel R, Ward E, Hao Y, Xu J, Thun MJ. Cancer statistics, 2009. CA Cancer J Clin 2009; 59: 225-49.

2. Parkin DM, Bray F, Ferlay J, Pisani P. Estimating the world cancer burden: Globocan 2000. Int J Cancer 2001; 94: 153-6.

3. La Vecchia C. Epidemiology of ovarian cancer: a summary review. Eur J Cancer Prev 2001; 10: 125-9.

4. Levi F, Lucchini F, Negri E, Boyle P, La Vecchia C. Cancer mortality in Europe, 1990-94, and an overview of trends from 1955 to 1994. Eur J Cancer 1999; 35: 1477-516.

5. Recine MA, Deavers MT, Middleton LP, Silva EG, Malpica A. Serous carcinoma of the ovary and peritoneum with metastases to the breast and axillary lymph nodes: a potential pitfall. Am J Surg Pathol 2004, 28: 1646-51.

6. Moore DH, Wilson DK, Hurleau JA, Look KY, Stehman FB, Sutton GP. Gynecologic cancers metastatic to breast. J Am Coll Surg 1998; 187: 858-61.

7. Karam AK, Stempel M, Barakat RR, Morrow M, Gernignani ML. Patients with a history of epithelial ovarian cancer presenting with a breast and/or axillary mass. Gynecol Oncol 2009; 112: 490-5.

8. Ben-Baruch G, Sivan E, Moran O, Rizel S, Menczer J, Seidman DS. Primary peritoneal serous papillary carcinoma: a study of 25 cases and comparison with stage III-IV ovarian papillary serous carcinoma. Gynecol Oncol 1996; 60: 393-6.

9. Moreira AL, Yao J, Waisman J, Cangiarella JF. Metastatic borderline papillary ovarian tumor in an intramammary lymph node. Breast 2002; 8: 309-10.

10. Micha JP, Goldstein BH, Epstein HD, Rettenmaier MA, Brown JV. Ovarian cancer metastatic to breast. Gynecol Oncol 2006; 102: 386-90.

11. Martel J, Roux JJ, Treilleux I, Bouchet P, Froger X, Mechin-Cretinon I. Breast metastases of an ovarian adenocarcinoma. Ann Dermatol Venereol 2003; 130: 623-5.

12. Kayikcioglu F, Boran N, Ayhan A, Güler N. Inflammatory breast metastasis of ovarian cancer: a case report. Gynecol Oncol 2001; 83: 613-6.

13. Zhao C, Bratthauer GL, Barner R, Vang R. Diagnostic utility of WT1 immunostaining in ovarian sertoli cell tumor. Am J Surg Pathol 2007; 31: 1378-86.

14. Yamamoto S, Tsuda H, Kita T, Maekawa K, Fujii K, Kudoh K, Furuya K, Tamai S, Inazawa J, Matsubara O. Clinicopathological significance of WT1 expression in ovarian cancer: a possible accelerator of tumor progression in serous adenocarcinoma. Virchows Arch 2007; 451: 27-35.

15. Tornos C, Soslow R, Chen S, Akram M, Hummer DJ, Abu-Rustum N, Nortan L, Tan LK. Expression of WT1, CA 125, and GCDFP-15 as useful markers in the differential diagnosis of primary ovarian carcinomas versus metastatic breast cancer to the ovary. Am J Surg Pathol 2006; 30: 140.

16. Fritzsche FR, Thomas A, Winzer KJ, Beyer B, Dankof A, Bellach J, Dahl E, Dietel M, Kristiansen G. Co-expression and prognostic value of gross cystic disease fluid protein 15 and mammoglobin in primary breast cancer. Histol Histopathol 2007; 22: 1221-30.

17. Kanner WA, Galgano MT, Stoler MH, Mills SE, Atkins KA. Distinguishing breast carcinoma from Müllerian serous carcinoma with mammoglobin and mesothelin. Int J Gynecol Pathol 2008; 27: 491-5.

18. Chia SY, Thike AA, Cheok PY, Tan PH. Utility of mammaglobin and gross cystic disease fluid protein-15 (GCDFP-15) in confirming a breast origin for recurrent tumors. Breast 2010; 19: 355-9.

\section{Address for correspondence}

\section{Dr. Lutfiye Demir}

Izmir Ataturk Training and Research Hospital

Clinic of Medical Oncology

tel. +90232 24444 44/ 2923

tel./fax +902322434443

e-mail: demir.lutfiye77@gmail.com

\section{Submitted: 8.07.2011 \\ Accepted: $\quad$ 13.11.2011}

